\title{
Place in therapy of innovative drugs in multiple myeloma in 2021 and 2023 according to an expert panel Delphi consensus
}

\author{
Boccadoro Mario ${ }^{1}$, Berto Patrizia², Bringhen Sara ${ }^{1}$, Zamagni Elena ${ }^{3}$, Tosi Patrizia ${ }^{4}$, Cascavilla Nicola ${ }^{5}$, Giuliani Nicola ${ }^{6}$, \\ Mannina Donato ${ }^{7}$, Zambello Renato ${ }^{8}$, Patriarca Francesca ${ }^{9}$, Montefusco Vittorio ${ }^{10}$, Grasso Mariella ${ }^{11}$, \\ Di Raimondo Francesco ${ }^{12}$, Offidani Massimo ${ }^{13}$, Petrucci Maria Teresa ${ }^{14}$, Musto Pellegrino ${ }^{15}$

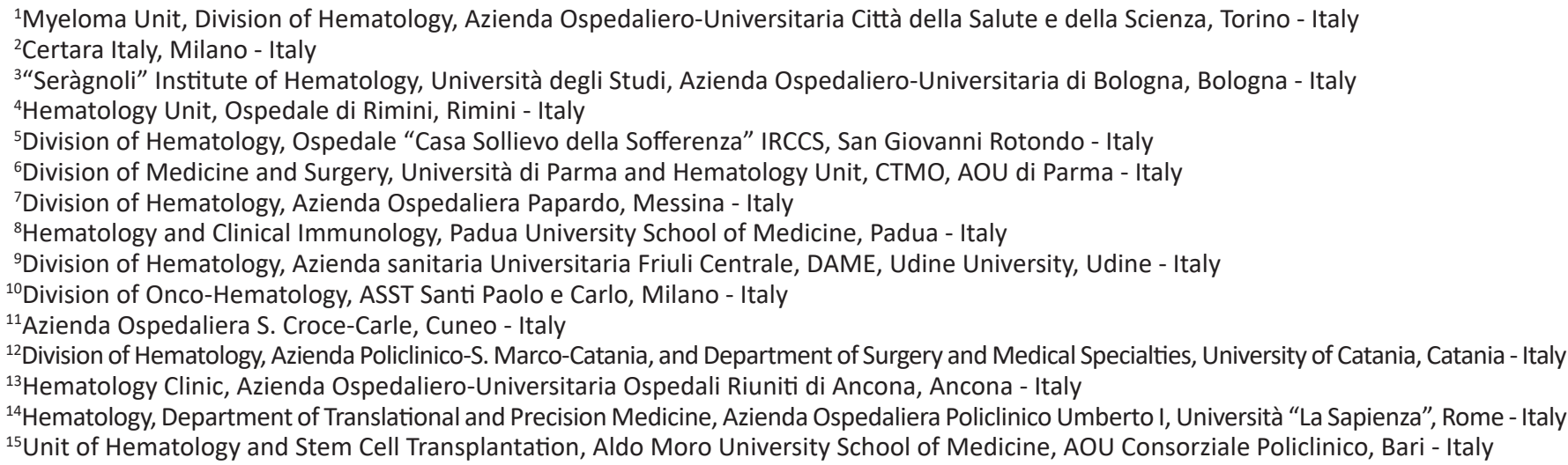

\begin{abstract}
Introduction: The objective of this study was to understand the potential use of single agents and drug combinations in multiple myeloma (MM) across treatment lines in the years 2021 and 2023.

Methods: The method used was Delphi Panel Method survey, administered to European Myeloma Network (EMN) Italy Working Group centres. Future treatments were identified assessing all available web-based information sources, including therapies (single drugs or combinations) with strong evidence of efficacy, likely to be on the Italian market in 2021 and 2023. Participants were asked to report on the likelihood of prescription for MM therapies, across treatment lines.

Results: Across the 15 centres taking part in the survey, about 890 patients per year are forecasted to receive a new diagnosis of MM. In 2021, the Panel forecasted $66 \%$ of $1 \mathrm{~L}-\mathrm{TE}$ (transplant eligible) patients will be treated with bortezomib-thalidomide-dexamethasone (VTD) and 32\% of patients with daratumumab-bortezomibthalidomide-dexamethasone (DVTd), with a substantial decrease of VTD (15\%) and a marked increase of DVTd (81\%) forecasted for 2023. The $2 \mathrm{~L}$ and $3 \mathrm{~L}$ R(lenalidomide)-based combination treatments are expected to drop and will likely be substituted by a steep increase in P(pomalidomide)-based regimes (from $7 \%$ to $23 \%$ ). On the contrary, in $3 \mathrm{~L}$ treatment, all combination therapies (with the exception of IsaPd - isatuximab-pomalidomidedexamethasone) are expected to lose market share in favour of the most recent new therapies.

Conclusions: Expert Panel agrees that many different new drugs and combinations will be used in MM, with different mechanisms of action, both at diagnosis and in subsequent phases of the disease, with a corresponding decline of the drugs currently used.
\end{abstract}

Keywords: Delphi Panel, European Myeloma Network, Monoclonal antibodies, Multiple myeloma

Received: February 17, 2021

Accepted: May 28, 2021

Published online: July 17, 2021

This article includes supplementary materials

Corresponding author:

Prof. Mario Boccadoro

Myeloma Unit, Division of Hematology

Azienda Ospedaliero-Universitaria Città della Salute e della Scienza

Via Genova 3

10126, Torino - Italy

mario.boccadoro@unito.it

\section{Introduction}

Multiple myeloma (MM) represents about $1 \%$ of all cancers and $10 \%$ of all haematological malignancies. In Italy, $\mathrm{MM}$ represents $1.3 \%$ of all cancers diagnosed in women and $1.2 \%$ in men, with an incidence of, respectively, 8.1 and 9.5 cases/100,000 per year. Estimates indicate a total of 2,098 new cases in women and 2,315 in men every year, with a homogeneous distribution among regions and a stable incidence over time, while mortality is slightly decreasing (1).

Most MM patients respond to the therapy, although only a very small fraction (5-10\%) are cured. The remaining 
eventually relapse after different remission durations, according to the biology of the disease (high or low risk) and the treatment. The majority of patients then receive a second line (2L) of treatment, usually achieving an inferior response compared to the previous line and with a shorter remission. A significant proportion of patients still receive a third line (3L), a reduced percentage a fourth and a fifth one (2).

Therapy of MM has remarkably improved over the past half century. For about three decades since the mid-1960s, alkylating agents, melphalan and cyclophosphamide, often combined with corticosteroids, were considered standard therapy (3). Starting in the 1990 s, autologous stem cell transplantation (ASCT) has been introduced for patients younger than 65 years, with a significant survival improvement. In the late 1990s, the immunomodulatory drug thalidomide was added to the traditional armamentarium, followed by its analogue, lenalidomide, in 2005 and pomalidomide in 2013 (3). Bortezomib was the first proteasome inhibitor to be evaluated for the treatment of MM in 2003, subsequently followed by carfilzomib and ixazomib, further increasing the treatment options for MM patients. In 2015, the Food and Drug Administration (FDA) approved two monoclonal antibodies (MoAb), daratumumab and elotuzumab, both targeting glycoproteins found on the surface of MM cells, CD38 and SLAMF7, respectively. Another anti-CD38 MoAb, isatuximab, was approved by the FDA and by the European Medicines Agency (EMA) in 2020, for relapsed/refractory MM (RRMM) $(3,4)$.

Many other new molecules and immunotherapies are showing promising antimyeloma activity in phase 1 and phase 2 protocols in patients who received several lines of therapies and were resistant to most of the previous drugs used (penta-refractory), such as selinexor, venetoclax or immunotherapy with CAR-T cells directed against B-cell maturation antigen (BCMA) plasma cell surface antigen (5). Phase 3 trials are being planned and these new drugs and strategies will be probably approved by Regulatory Agencies and will become available in the next few years.

In this quite complex scenario, two basic and relevant aspects should be taken in account. First, every new agent active in $\mathrm{MM}$ will certainly not be able to eradicate the disease by itself, but it will be used in a sequence/combination with other drugs. Biological studies can help define the best partner or partners (combination therapies or Combo) but only phase 3 studies will show the best efficacy/toxicity ratio. Second, all new agents are rarely compared in large, headto-head clinical studies, and only indirect cross-trial comparisons are possible, with all the limitations related to the different patient selection and drug administration.

Literature review suggests there is an information gap as to which drugs/combination therapies will actually be prescribed in first line (1L), $2 \mathrm{~L}$ and $3 \mathrm{~L}$ treatments of $\mathrm{MM}$, in Italy in the near future, especially considering the huge number of new single-drug treatments (SDTs) and combination therapies expected to be launched in the next years.

In order to fill this gap, we designed a survey to be administered through the Delphi Panel Method to a sample of Italian clinical centres belonging to the Working Group of the European Myeloma Network (EMN) Italy, representative of the Italian MM treatment setting.
The Delphi Panel Method has been applied for over 30 years in the clinical healthcare research environment as a tool for conducting structured and efficient surveys on clinical topics for which clear, univocal, evidence-based information is missing, or in situations in which different orientations are possible $(6,7)$. This technique is designed as a group communication process, aimed at achieving a convergence of opinions on a specific real-world issue (8). The methodology is applied by questioning a group of subjects on a certain topic, evaluating similarities and disparities in their answers, with the ultimate goal of reaching expert consensus (6).

Some distinguishing features of the Delphi technique are the anonymity of respondents, a controlled feedback process and the suitability of a variety of statistical analysis techniques to interpret the data (8). These characteristics are designed to offset the shortcomings of conventional means of pooling opinions, obtained from group interaction (i.e. influences of dominant individuals, noise and group pressure for conformity); in fact, subject anonymity can reduce the effects of dominant individuals, which often is a concern when using group-based processes to collect and synthesize information (9). Additionally, the issue of confidentiality is facilitated by geographic dispersion of the subjects, as well as the use of electronic communication such as e-mails to solicit and exchange information (8).

In line with the Delphi methodology, the objective of this project was to collect data on the predicted, future use of SDT and combination in all treatment lines of MM, in the years 2021 and 2023, in Italy.

\section{Methods}

The experts participating in this survey represent the different Italian haematological centres, geographically well distributed across the country, and are members of the EMN Italy Working Group. EMN is a non-profit organization created in 2005 to increase the understanding of the disease, and to produce effective research projects and clinical trials. Various countries are in fact represented within this large cooperation network, and the EMN is an extensive platform that can be used by European investigators to start new trials and collaborative initiatives. As for its structure, the EMN headquarters are located in Rotterdam and the EMN central office closely collaborates with the data centre and Italian branch of the network, namely the EMN Research Italy. EMN at present sponsors several clinical trials including newer drugs (see EMN website).

Our survey adopted a "modified" Delphi Panel technique, based on a pre-defined structured questionnaire (Delphi Panel Data Collection Form), administered to a number of Italian myeloma experts who filled and returned the questionnaire $(8,10-12)$. After the first round of response, a second Delphi round was planned to validate deviance from mean values, based on direct e-mail exchange with each respondent. Confidentiality and freedom of expression was maintained by interaction of each respondent with an external observer.

The questionnaire was developed around the identification of all SDT or combinations that are expected to be available and reimbursed by or within 2021 and 2023, in each of the following lines of treatment: $1 \mathrm{~L}$, split between ASCT-eligible (1L-TE) and ASCT non-eligible (1L-TNE); 2L; 3L (and further lines, 3L+). 
In our study, identification of future treatments was based on thorough scrutiny of all available web-based information sources - including the websites of European Medicines Agency (EMA), Agenzia Italiana del Farmaco (AIFA), Associazione Italiana Oncologia Medica (AIOM), Associazione Italiana di Ematologia e Oncologia Pediatrica (AIEOP) and Società Italiana di Ematologia (SIE) - validated with reports and publications from the most recent congresses and events, as well as with the pipelines of the relevant pharmaceutical companies. Final selection comprised only therapies (SDT or Combo) for which strong evidence of efficacy was available, based at least on interim analysis of pivotal, approval-aimed trials, as disclosed by the marketing authorization holder (MAH), justifying reasonable expectation of availability on the Italian market, in the timeframe of our analysis, i.e. years 2021 and 2023. The list of SDT and Combo, by line of treatment, investigated in the questionnaire is reported in Table I.

Participants were asked to answer two sets of questions: (i) an epidemiology section, aimed at collecting general information on the number of incidents patients typically managed at the respondent's centre per year and their distribution among the different lines of treatment, and (ii) the forecasting section, in which each centre was asked to distribute 100 percentage points to SDT or combination more likely to be prescribed in 2021 and 2023 for treatment of MM, across treatment lines. A copy of the questionnaire is reported in the Supplementary material (Delphi Panel Data Collection Form, in Italian).

Statistical analysis was performed by standard statistical methods, calculating means, standard deviation, minimum and maximum values. After the first round of questionnaires, an external observer contacted each centre to validate discrepancies with calculated mean values in order to get confirmation from each respondent.

\section{Results}

Fifteen centres took part in the survey, being geographically well distributed across northern, central and southern Italy's regions. Summary of epidemiology data is presented in Figure 1 . Across the 15 participating centres 890 patients per year are newly diagnosed with MM: of these $94 \%(n=838)$ of patients undergo $1 \mathrm{~L}$ treatment for MM; $42 \%(n=349)$ of them are TE; $58 \%(n=489)$ are considered TNE; overall, $79 \%$ ( $n=703$ ) of patients undergo $2 \mathrm{~L}$ treatment for $\mathrm{MM}$ and $44 \%$ $(\mathrm{N}=395)$ undergo $3 \mathrm{~L}+$ treatments (Fig. 1 ).

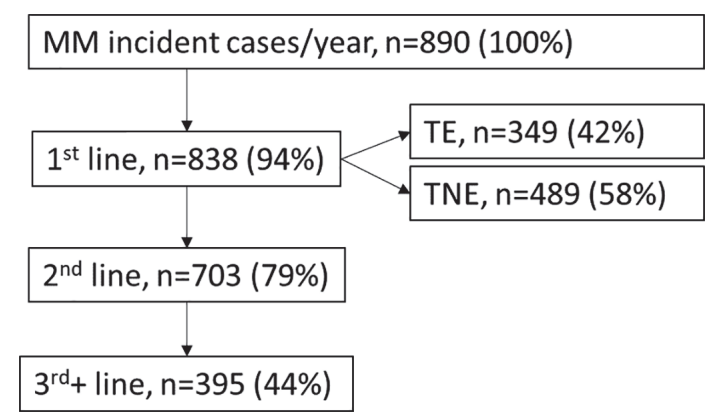

Fig. 1 - Distribution of incident MM patients across treatment lines (N). $\mathrm{MM}=$ multiple myeloma; $\mathrm{TE}=$ transplant eligible; $\mathrm{TNE}=$ transplant non-eligible.
TABLE I - Single-drug treatments and combination drug schemes by line of treatment in $\mathrm{MM}$

\begin{tabular}{lc}
\hline Acronym & Drug/Combination name \\
\hline 1st line Transplant Eligible (1L-TE) \\
\hline VTd & bortezomib-thalidomide-dex \\
DVTd & daratumumab-bortezomib-dex-thalidomide
\end{tabular}

Maintenance treatment

Ixa ixazomib maintenance (2023)

$\mathrm{R} \quad$ lenalidomide maintenance

\begin{tabular}{|c|c|}
\hline \multicolumn{2}{|c|}{ 1st line Transplant Non-Eligible (1L-TNE) } \\
\hline $\operatorname{Rd}$ & lenalidomide-dex \\
\hline VMP & bortezomib-melphalan-prednisone \\
\hline MPT & melphalan-prednisone-thalidomide \\
\hline DRd & daratumumab-lenalidomide-dex \\
\hline DVMP & $\begin{array}{l}\text { daratumumab-bortezomib-melphalan- } \\
\text { prednisone }\end{array}$ \\
\hline VRd & bortezomib-lenalidomide-dex \\
\hline \multicolumn{2}{|c|}{ Maintenance treatment } \\
\hline Ixa & ixazomib maintenance (2023) \\
\hline \multicolumn{2}{|c|}{ 2nd Line (2L) } \\
\hline $\mathrm{Rd}$ & lenalidomide-dex \\
\hline DRd & daratumumab-lenalidomide-dex \\
\hline KRd & carfilzomib-lenalidomide-dex \\
\hline DVd & daratumumab-bortezomib-dex \\
\hline ERd & elotuzumab-lenalidomide-dex \\
\hline $\mathrm{Kd}$ & carfilzomib-dex \\
\hline IxaRd & ixazomib-lenalidomide-dex (high risk) \\
\hline PVd & pomalidomide-bortezomib-dex \\
\hline DKd & daratumumab-carfilzomib-dex \\
\hline DPd & daratumumab-pomalidomide-dex \\
\hline IsaKd & isatuximab-carfilzomib-dex \\
\hline SVd & selinexor-bortezomib-dex \\
\hline
\end{tabular}

3rd (and further) Line (3L+)

$\mathrm{Pd} \quad$ pomalidomide-dex

DVd daratumumab-bortezomib-dex

DRd daratumumab-lenalidomide-dex

Rd lenalidomide-dex

Kd carfilzomib-dex

IxaRd ixazomib-lenalidomide-dex

KRd carfilzomib-lenalidomide-dex

EPd elotuzumab-pomalidomide-dex

IsaPd isatuximab-pomalidomide-dex

ide-cel/CAR-T Idecabtagene vicleucel (CD38-exposed)

anti-BCMA belantamab mafodotin (CD38-refractory)

Sd selinexor-dex (penta-refractory)

dex = dexamethasone; $\mathrm{MM}=$ multiple myeloma 
In 1 L-TE patients, the Panel expects that $66 \%$ of patients will be treated with the standard combination bortezomib-thalidomide-dexamethasone (VTD) and $32 \%$ of patients with daratumumab-bortezomib-thalidomide-dexamethasone (DVTd) in 2021; the Panel forecasted a substantial decrease of VTD to $15 \%$ and a marked increase of DVTd to $81 \%$ for 2023 (Fig. 2). In these patients, 1L maintenance treatment will be performed mainly ( $84 \%$ of patients) with lenalidomide (R) monotherapy in 2021, while in 2023 this frequency is expected to fall to $72 \%$, with $22 \%$ of patients potentially exposed to maintenance treatment with ixazomib (Ixa).

In 1 L-TNE patients, in 2021, the Panel expects $32 \%$ of patients will be treated with bortezomib-melphalan-prednisone (VMP); 26\% with lenalidomide-dexamethasone (Rd); $22 \%$ with daratumumab-lenalidomide-dexamethasone (DRd); $11 \%$ with daratumumab-bortezomib-melphalan-prednisone
(DVMP) and 8\% with bortezomib-lenalidomide-dexamethasone (VRd). For 2023, the most significant variation in this treatment line is a predicted substantial increase for DRd (41\%) and smaller increases for DVMP (19\%) and VRd (14\%) with considerable reductions for both $\mathrm{Rd}(13 \%)$ and VMP (10\%) (Fig. 3). Most of the treatments in TNE are until progression, thus a specific question on maintenance with Ixa yielded $100 \%$ negative answers.

As expected, according to the expert Panel, quite a number of options will be available for $2 \mathrm{~L}$ treatment of $\mathrm{MM}$, that is, RRMM, with several new SDT in 2023 and various combinations launched between 2021 and 2023. In particular, pomalidomide-bortezomib-dexamethasone (PVd) and daratumumabcarfilzomib-dexamethasone (DKd) will be probably launched during 2021; daratumumab-pomalidomide-dexamethasone (DPd) and selinexor-bortezomib-dexamethasone (SVd) in
1 LINE - TE

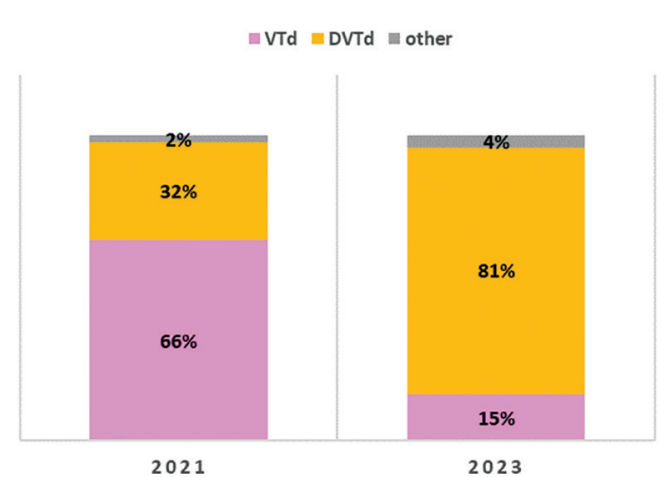

2021
1 LINE TE - MAINTENANCE

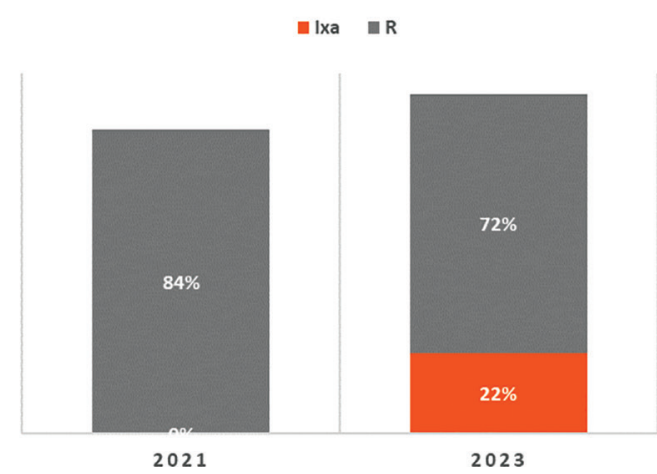

Fig. 2 - Distribution of MM treatments in $1 \mathrm{~L}-\mathrm{TE}$.

$\mathrm{MM}=$ multiple myeloma; $\mathrm{TE}=$ transplant eligible. Abbreviations for single-drug and combination treatment schemes are reported in Table I.

\section{LINE - TNE}

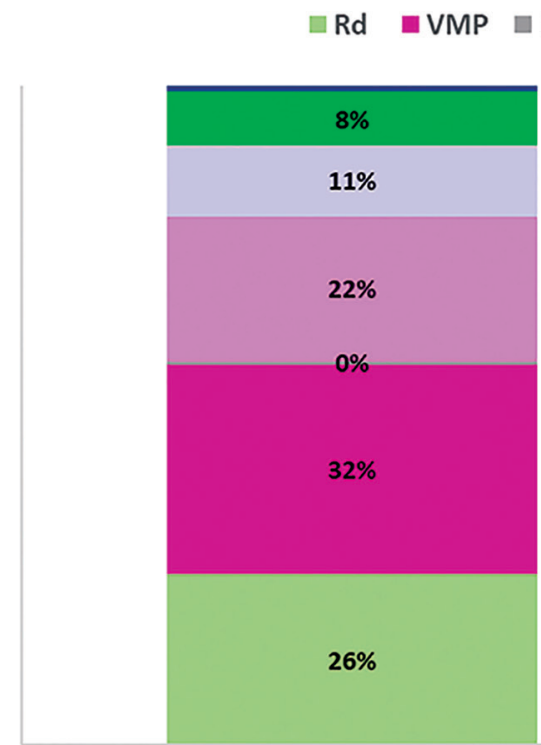

2021
MPT $\square$ DRd $\square$ DVMP $\square$ VRd $\square$ other
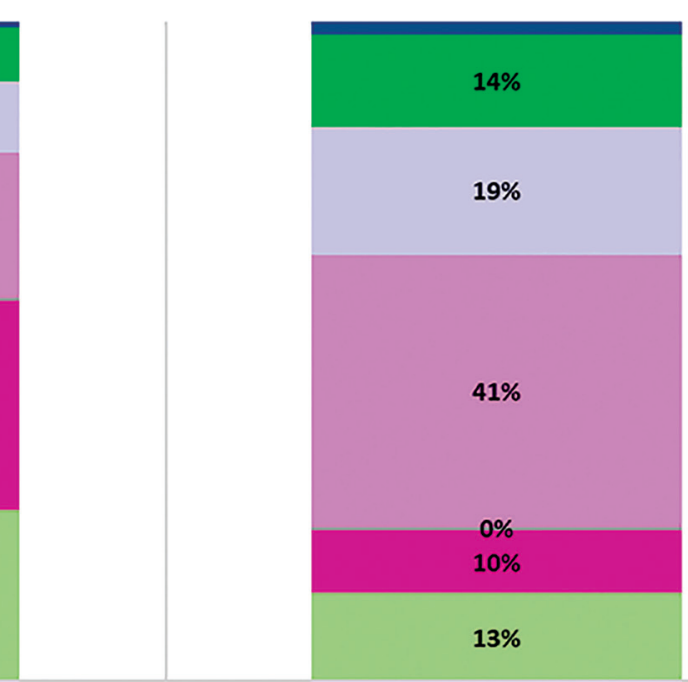

20
Fig. 3 - Distribution of MM treatments in 1L-TNE. $\mathrm{MM}=$ multiple myeloma; TNE = transplant non-eligible. Abbreviations for single-drug and combination treatment schemes are reported in Table I. 
2022 and isatuximab-carfilzomib-dexamethasone (IsaKd) in 2023 (Fig. 4). IxaRd, PVd and DKd are all expected to gain market shares both in 2021 and 2023: IxaRd from 4\% in 2021 to $6 \%$ in 2023; DKd 6\% to $11 \%$ and PVd $7 \%$ to $9 \%$. DPd and IsaKd are expected to take, respectively, $14 \%$ and $8 \%$ of the market in 2023, with SVd left in a marginal position (only $2 \%$ in 2023). As an effect of all these new entries, the two combination therapies collectively representing more than $50 \%$ of the market in 2021, DRd and carfilzomib-lenalidomide-dexamethasone (KRd) with respectively $34 \%$ and $19 \%$ market share in 2021 , would both be sized down to $22 \%$ and $11 \%$, respectively, in 2023.

Similar to $2 \mathrm{~L}, 3 \mathrm{~L}$ treatment of RRMM will be characterized by a variety of options in the next few years, as described in Figure 5, with a total of 12 combination therapies present on the Italian market in 2021 and 2023. Five new combination therapies are expected to be launched during 2021: elotuzumab-pomalidomide-dexamethasone (EPd); isatuximab-pomalidomide-dexamethasone (IsaPd); idecabtagene vicleucel in CD38-exposed patients (ide-cel/CAR-T); belantamab mafodotin, anti-BCMA in CD38-refractory patients and selinexor-dexamethasone, in penta-refractory patients $(\mathrm{Sd})$. As seen in $2 \mathrm{~L}$, also in the $3 \mathrm{~L}$ setting the use of combination therapies most likely adopted in 2021 is expected to significantly decrease afterwards. In particular, according to the Panel, Pd will cover $19 \%$ of treatments in Italy, DRd $13 \%$, DVd 9\%, Kd 9\%, IxaRd 8\%, and they will be superseded by the newest combination therapies; and SDT, specifically IsaPd, anti-BCMA and ide-cel/CAR-T, representing 16\%, 15\% and 9\% of expected treatments, respectively.

An analysis can be drawn from the aggregation of all combination therapies containing the most frequently associated agents: daratumumab, pomalidomide, lenalidomide and carfilzomib. Figure 6 shows the expected frequency of prescription for all combination therapies containing each of these four drugs. For example, for daratumumab in $2 \mathrm{~L}$, DRd, DVd, DKd and DPd combination therapies are included. Similarly, for pomalidomide in 2L, PVd and DPd are included. Across combination treatments aggregated around the four drugs, some fall under more than one of the four drug categories and are necessarily counted more than once. Nevertheless, the scope of this sub-analysis is mainly to highlight the macro trends. The use of $2 \mathrm{~L} \mathrm{R}$-based combination treatments will drop from $67 \%$ in 2021 to $44 \%$ in 2023; and they will also decrease in $3 \mathrm{~L}$ from $31 \%$ in 2021 to $21 \%$ in 2023. According to the Panel, $2 \mathrm{~L}$ R-based treatment schemes will likely be substituted by a steep increase in P-based regimes (from $7 \%$ to $23 \%$ ) and a smaller increase in D-based regimes (53\% to

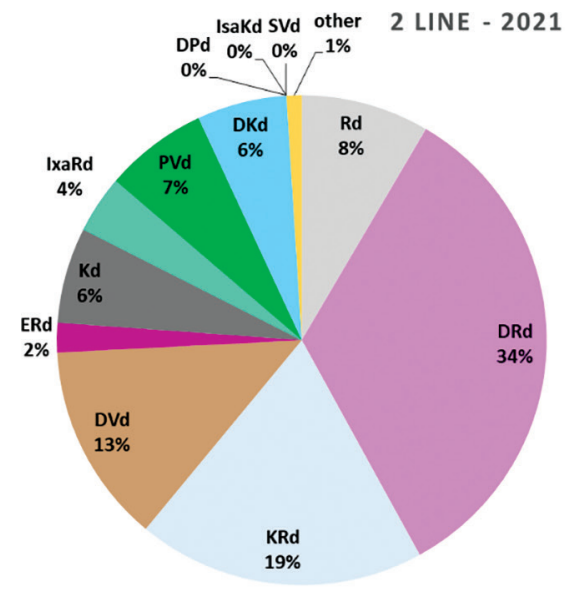

3 LINE - 2021

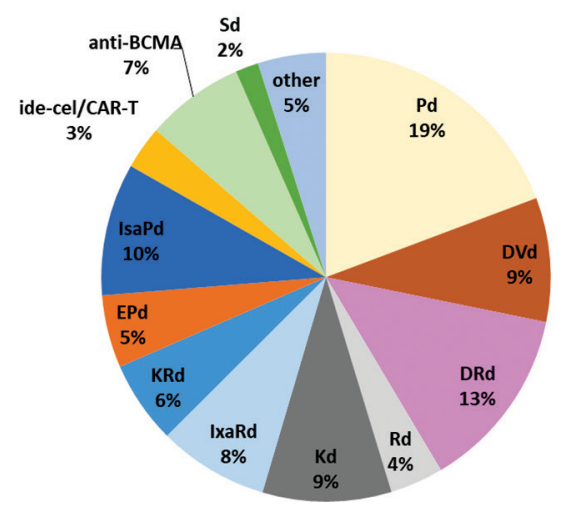

2 LINE - 2023

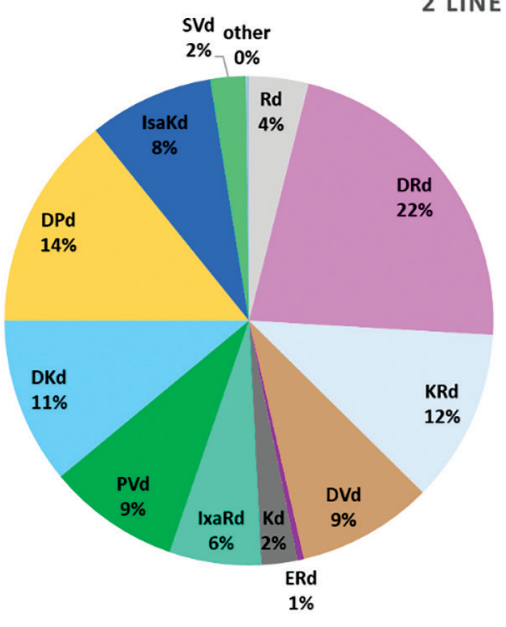

3 LINE - 2023

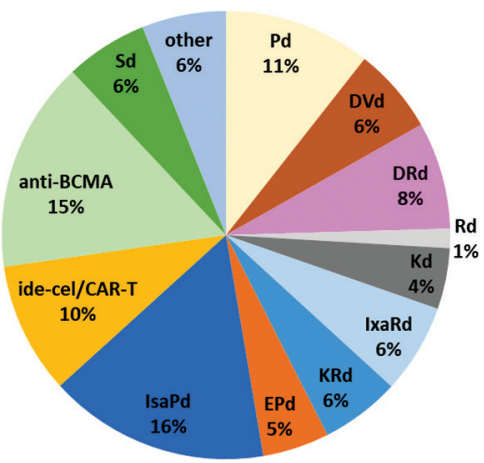

Fig. 4 - Distribution of RRMM treatments in 2nd line of treatment.

RRMM = relapsed refractory multiple myeloma. Abbreviations for single-drug and combination treatment schemes are reported in Table I. 


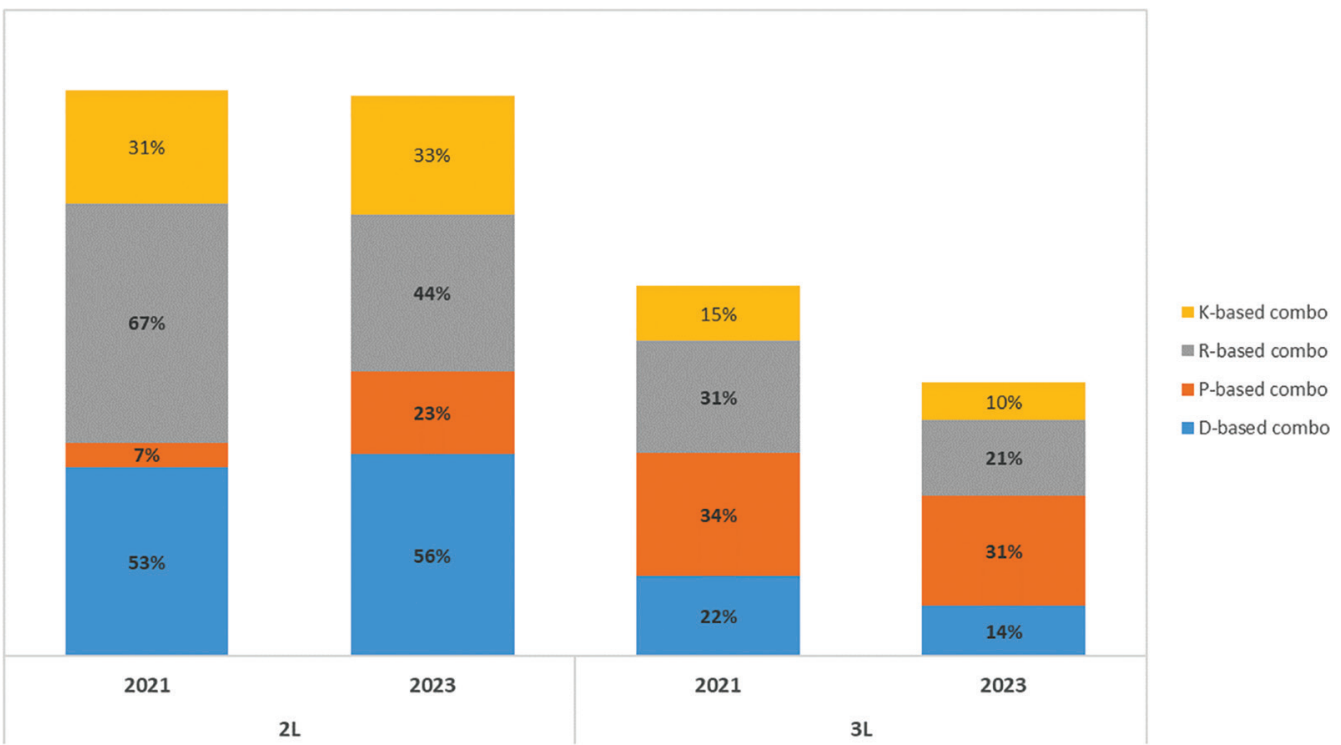

Fig. 6 - Distribution of MM treatments in 2 nd, 3 rd and subsequent line of treatment in RRMM.

D, daratumumab; K, carfilzomib; $P$, pomalidomide; $R$, lenalidomide; $R R M M=$ relapsed refractory multiple myeloma.

$56 \%)$. On the contrary, in $3 \mathrm{~L}$ treatment, all these combination therapies (with the exception of IsaPd) are expected to lose market shares in favour of the most recent new entries: ide-cel/CAR-T, anti-BCMA and Sd.

\section{Conclusion}

In this paper a myeloma expert Panel aimed to forecast the treatments that MM patients will receive in 2021 and 2023 at diagnosis, at first, second and third relapse in Italy. To the best of our knowledge, this is the first analysis to investigate the positioning of various drugs and combination in 1 and 3 years from now, using a structured method of putting together perspectives of several haematology experts, by means of the Delphi methodology.

Several new myeloma treatment drugs have been introduced in the last few years, many more will be available in the years to come, leading to an incredibly high number of possible combination therapies. Unfortunately, future predictions of MM treatments are not available in the literature.

For MM patients at diagnosis, our expert Panel is in favour of treatment combination therapies using anti-CD38 MoAb both in TE and NTE patients; DRd and DVTD will increasingly be adopted both in 2021 and in 2013. Their constant increase probably reflects a delay in their actual availability, after regulatory approval: DRd is expected to increase from $22 \%$ to $41 \%$ in 2021 and 2023, DVMP from 11\% to 19\% in 2021 and 2023, DVTD from 32\% to $81 \%$ in 2021 and 2023. Apparently, the use of triplet or quadruplet combination therapies at diagnosis - i.e. those that are expected to be the most effective - is the most widely accepted and sensible strategy. Thus, the possible appearance of resistant clones caused by using active induction drugs at diagnosis seems not to impact treatment decision: using active agents upfront to prolong progression-free survival is probably considered a priority versus delaying their use for later stages at relapse.

At first and second relapse, we hypothesize that at least nine new drugs or combination therapies will be made available in 2021 and 2023; thus, the choice for the treating haematologist will be more complex. However, looking at the overall results of our survey, we can conclude that our experts are in favour of adopting new drugs with different mechanisms of action in 2021 and in 2023. The Panel believes that there will be room for the novel anti-BCMA immunotherapies, both immunoconjugate belantamab and CAR-T cells. Consequently, a significant decrease in the standard treatments based on lenalidomide, pomalidomide and anti-CD38 MoAb is expected. All the new drugs entering the market are supported by a clear statistically significant superiority over the standard of care. However, once approved, some drugs are immediately widely used, while others seem to be scarcely adopted or neglected. This is not surprising considering the patient selection that can occur in similar trials, the choice of the control arm and finally the toxicity profile. There are no head-to-head trials using homogeneous inclusion criteria and identical control arms. Strong survival differences certainly influence the physician's choice, but when it comes to trials showing similar results, the balance between efficacy and toxicity is basically left to the clinicians' judgement and personal experience. This is why we asked myeloma experts who are already using these new effective drugs in the clinical trial context - which will be made available in the coming years - to share their experience and to bet on future drugs approval, reimbursement, likelihood to be used and their positioning in the various lines of therapy.

By estimating the market shares of existing drugs alongside the new available drugs in $1 \mathrm{~L}-\mathrm{TE}, 1 \mathrm{~L}-\mathrm{NTE}, 2 \mathrm{~L}$ and $3 \mathrm{~L}+$, the Panel has intrinsically considered the effect of new drugs and combination therapies not only on the specific treatment line but also on the global effect across all treatment lines, thus providing a comprehensive picture of how the whole MM will evolve in the near future.

Every drug positioning evaluation inevitably carries some bias. In the present evaluation, we are including haematologists prescribing drugs for the various phases of $\mathrm{MM}$ treatment and certainly only partially representative of the highly 
heterogeneous Italian landscape, including small, large hospitals and academic institutions. We only considered reference centres and physicians for this specific disease, collaborating with the EMN Italy and the large European networks, who are aware of all the new treatments and who mostly also participate in clinical trials with innovative drugs. Conversely, it would have been hardly feasible to conduct the same analysis with prescribing physicians, non-MM experts, representative of the various Italian hospitals, since misleading conclusions could have been drawn.

In conclusion, this expert Panel tried to shed some light on a very complex matter, where data about future predictions are lacking. Although a true mathematical model could not be applied, an alternative reliable and useful approach was used, that is, the Delphi technique. Basically, our expert Panel members believe that in MM there is room for many new drugs, with different mechanisms of action, both at diagnosis and in subsequent phases of the disease, with a corresponding decline of the drugs currently used.

\section{Acknowledgement}

Medical writing support was provided by Certara Italy and supported by Sanofi.

\section{Contributorship statement}

$M B$ and $P B$ developed the initial version of the questionnaire; $P B$ analysed the data; $M B, S B, E Z, P T, N C, N G, D M, R Z$, FP, VM, MG, FDR, MO, MTP, PM filled the two Delphi Panel rounds and provided revisions of the manuscript.

\section{Disclosures}

Conflict of interest: M.B. has received honoraria from Sanofi, Celgene, Amgen, Janssen, Novartis, Bristol-Myers Squibb and AbbVie; has served on the advisory boards for Janssen and GSK; has received research funding from Sanofi, Celgene, Amgen, Janssen, Novartis, Bristol-Myers Squibb and Mundipharma. P.B. is a consultant of Certara Italy. S.B. has received honoraria from Celgene, Amgen and Janssen, and Bristol-Myers Squibb; has served on the advisory boards for Celgene, Amgen, Janssen and Karyopharm; has received consultancy fees from Janssen and Takeda. E.Z. has received honoraria from and served on the advisory board for Janssen, BMS, Takeda, Sanofi, Amgen. N.C. has received honoraria from Takeda, Pfizer, Janssen Pharmaceutical, Bristol Myers Squibb, Amgen, Celgene, Novartis. N.G. has received research grants from Celgene, Janssen Pharmaceutical; clinical trial sponsorship from Janssen Pharmaceutical, Millennium Pharmaceutical, GSK; served on the advisory boards for Celgene, Takeda, Janssen Pharmaceutical; and congress fee from Janssen Pharmaceutical, Celgene, Bristol-Myers
Squibb. R.Z. has served on the advisory board for Janssen, Celgene, GSK. F.P. has served on the advisory board for Celgene/ Bristol-Myers Squibb, Janssen, GSK. V.M. has received honoraria and travel grants from Janssen, Celgene, Bristol-Myers Squibb, Amgen, Takeda. F.D.R. has received honoraria from and served on the advisory boards for Janssen, Celgene, Amgen, Takeda, GSK. M.O. has received honoraria from and served on the advisory boards for Amgen, BMS, Celgene, Janssen, Sanofi, Takeda. M.T.P. has received honoraria from and served on the advisory board for Celgene, Janssen-Cilag; Bristol Myers Squibb, Takeda, Amgen, Sanofi, GSK. P.M. has received personal fees and/or served as a member of advisory boards from/of Amgen, Novartis, BMS, Celgene, Janssen, Sanofi, Gilead, Abbvie, GSK, Jazz, Takeda. The other authors declare no competing conflicts of interest.

Financial support: this study was funded by Sanofi.

\section{References}

1. AIOM Associazione Italiana Oncologia Medica. Linee Guida Mieloma. Edizione 2017 Online Online (accessed November 2020).

2. Kumar SK, Rajkumar V, Kyle RA, et al. Multiple myeloma. Nat Rev Dis Primers. 2017;3(1):17046. CrossRef PubMed

3. Abramson HN. B-cell maturation antigen (BCMA) as a target for new drug development in relapsed and/or refractory multiple myeloma. Int J Mol Sci. 2020;21(15):5192. CrossRef PubMed

4. European Medicines Agency Sarclisa - isatuximab. Online Online (accessed November 2020).

5. Shah N, Chari A, Scott E, Mezzi K, Usmani SZ. B-cell maturation antigen (BCMA) in multiple myeloma: rationale for targeting and current therapeutic approaches. Leukemia. 2020;34(4):985-1005. CrossRef PubMed

6. Acone B, Urbani A. The Delphi Method: The methodology and its application. JHA. 2018;S1(S1):9-14. CrossRef

7. Trevelyan EG, Robinson N. Delphi methodology in health research: how to do it? Eur J Integr Med. 2015;7(4):423-428. $\underline{\text { CrossRef }}$

8. Hsu CC, Sandford BA. The Delphi Technique: making sense of consensus. Pract Assess Res Eval. 2007;12(10). CrossRef (accessed November2020).

9. Dalkey N, Helmer O. An experimental application of the Delphi Method to the use of experts. Manage Sci. 1963;9(3):458-467. CrossRef

10. Kerlinger FN. Foundations of behavioural research. New York: Holt, Rinehart, and Winston, Inc 1973.

11. Linstone HA, Turoff M. The Delphi method: techniques and applications. Reading, MA: Addison-Wesley Publishing Company 1975.

12. Dalkey NC, Rourke DL. Experimental assessment of Delphi procedures with group value judgments. In: Dalkey NC, Rourke DL, Lewis R, et al, eds. Studies in the quality of life: Delphi and decision-making. Lexington, MA: Lexington Books 1972; 55-83. 